Revue internationale de l'économie sociale

Recma

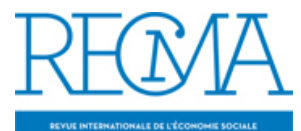

\title{
Questionner la responsabilité
}

\section{Le comité organisateur du colloque}

Numéro 304, mai 2007

URI : https://id.erudit.org/iderudit/1021528ar

DOI : https://doi.org/10.7202/1021528ar

Aller au sommaire du numéro

Éditeur(s)

Association Recma

ISSN

1626-1682 (imprimé)

2261-2599 (numérique)

Découvrir la revue

Citer ce document

Le comité organisateur du colloque (2007). Questionner la responsabilité.

Revue internationale de l'économie sociale, (304), 9-12.

https://doi.org/10.7202/1021528ar d'utilisation que vous pouvez consulter en ligne.

https://apropos.erudit.org/fr/usagers/politique-dutilisation/ 


\section{QUESTIONNER LA RESPONSABILITÉ}

(1) Coorganisées par les universités Rennes 1 et Rennes 2, université Haute-Bretagne, les 24 et 25 mai 2007, à Rennes.

(2) Cf. liste des communications en encadré. 'économie sociale et solidaire face à ses responsabilités ", tel est le thème des VII ${ }^{\text {es }}$ Rencontres internationales du Réseau interuniversitaire de l'économie sociale et solidaire ${ }^{(1)}$.

Face aux nouveaux défis d'aujourd'hui et à l'extension du concept de responsabilité sociale ou sociétale dans l'espace et le temps, les organisations de l'économie sociale et solidaire se retrouvent situées dans une position relativement paradoxale en fonction de leur stade de développement et/ou de leur projet et de leurs valeurs. En effet, elles sont à la fois acteur de référence et expérimentateur privilégié, à la fois en capacité de défricher, d'innover et de stimuler ce champ d'investissement, mais aussi en position de faire émerger et de médiatiser ces nouvelles demandes sociales, voire de les reformuler ou de les rejeter. Comment les organisations assument-elles ces responsabilités?

En proposant aux membres et contacts du réseau de se réunir autour de la thématique de la responsabilité, nous avions conscience de soulever une question dérangeante, pour ne pas dire provocatrice. Il est en effet surprenant de constater que, si la question de la responsabilité présente une grande proximité avec un ensemble de notions et de concepts liés au champ de l'économie sociale et solidaire, comme ceux d'éthique, de valeurs ou de repères, elle est en elle-même rarement interrogée. Sans doute semblet-elle aller de soi, relever de l'évidence. Or nous savons en tant que chercheurs que les notions reçues pour évidentes, et donc rarement questionnées ou remises en question, sont souvent celles qui méritent l'examen critique le plus approfondi. C'est à ce déficit de questionnement que nous avons voulu nous attaquer en choisissant ce thème de réflexion, qui a donné lieu à une trentaine de communications, dont plusieurs internationales ${ }^{(2)}$. Deux orientations fortes se dégagent à l'examen de ces différentes communications. Tout d'abord, loin de chercher à circonscrire la question de la responsabilité des organisations de l'économie sociale à des limites bien établies, elles développent un champ d'investigation qui semble sans frontières, concernant toutes les facettes de la vie sociale et tous les registres (juridique, éthique et politique). Cette extension tend d'un côté à conférer à l'économie sociale et solidaire une place centrale dans les différents processus formateurs d'une société: développement économique durable dans les territoires, maintien et dynamique du lien social, production d'innovations et de dispositifs socio-économiques répondant à l'émergence de nouveaux besoins, développement de nouveaux axes de performances pour les entreprises et les associations, extension du champ de la démocratie et de l'égalité. D'un autre côté, elle tend à estomper ce que l'on dénomme classiquement les spécificités de l'économie sociale et solidaire. 
Cette question des limites et des spécificités ressort également dans une autre perspective. L'on semble en effet confronté dans de nombreux champs d'activité à une convergence croissante du fonctionnement ou des régulations des structures de l'économie sociale et solidaire et du fonctionnement ou des régulations des entreprises classiques. Parallèlement, la montée en puissance des thèmes de la responsabilité sociale des entreprises ou de l'investissement socialement responsable vient aussi interpeller directement la prétention de ces structures à incarner une philosophie différente de l'activité économique. Ce double mouvement de convergence apparente appelle ainsi une démarche renouvelée de clarification, de justification et de démonstration de la spécificité des structures de l'économie sociale et solidaire. Les quatre communications sélectionnées pour publication à l'occasion de ce colloque sont représentatives de ces deux ensembles de problématiques. Ainsi, la communication de Gaël Henaff aborde le phénomène juridique de la banalisation du régime de responsabilité du dirigeant d'association. Le maintien du statut originel de la loi de 1901 s'est en effet accompagné d'une évolution de la jurisprudence faisant de plus en plus intervenir les principes de droit commun des sociétés pour traiter la question du statut des dirigeants d'association, ce qui conduit à assimiler des élus bénévoles aux dirigeants rémunérés des sociétés commerciales. On perçoit ici combien sous l'angle de cette responsabilité particulière s'efface aux yeux du droit l'un des éléments fondamentaux de la particularité de l'action désintéressée. L'auteur souligne ce faisant les insuffisances d'une analyse essentiellement contractuelle du fonctionnement des associations, ne reconnaissant pas les finalités spécifiques qui fondent leur personnalité morale. La communication de Pascal Glémain et de Marie-Thérèse Taupin traite sous l'angle des finances solidaires la question des rapports de l'économie sociale et solidaire avec les organisations financières marchandes. En effet, les institutions liées à ce qu'il est convenu de dénommer l'investissement socialement responsable (ISR) ont su identifier les différentes dimensions de leurs responsabilités: économique, sociale et environnementale. Cette posture d'explicitation conduit à confronter alors les organisations des finances solidaires à l'élucidation des critères et repères souvent implicites qui guident leurs pratiques. Parmi ceux-ci pourraient figurer l'approche du bien-être et l'amélioration de la compréhension des mécanismes économiques qui lient épargne et développement.

En parallèle à cette perspective, Cécile Lapenu et François Doligez se sont attaqués à la question de l'évaluation des actions des organismes de microcrédit. En effet, le rapprochement structurel marqué de ceux-ci avec le fonctionnement du marché bancaire et financier (développement des approches commerciales, renforcement de l'investissement privé et du partenariat bancaire) a comme contrepartie une obligation de justifier de la poursuite d'objectifs réellement sociétaux. L'opérationnalisation de cette évaluation n'est pas sans poser un certain nombre de problèmes et notamment du point de vue de ses finalités, selon que l'on considère que l'on poursuit des objectifs de pilotage ou des objectifs de notation à destination du public. 
Enfin, Florian Perret et Martino Nieddu s'interrogent sur la place des communautés liées à l'élaboration et à la diffusion des logiciels libres dans leur environnement stratégique. Si les logiciels libres s'élaborent à partir de pratiques coopératives non marchandes, s'établissant sur des valeurs de coopération et de partage des savoirs, ils ne s'en inscrivent pas moins dans des mécanismes de régulation du marché des logiciels. Dès lors, ces pratiques ont un impact sur des stratégies de marché confrontées à cette hybridation et modifient les conditions mêmes qui configurent la concurrence entre les entreprises.

Toutes ces réflexions conduisent donc à reconsidérer les liens qui unissent un champ souvent considéré comme autonome avec le cadre sociétal où il s'inscrit. La question centrale semble ainsi être l'identification des interactions complexes qui relient les organisations de l'économie sociale et solidaire avec leur champ d'action et leur espace de ressources. Si d'un certain point de vue, elles apparaissent de plus en plus dépendantes de ressources que l'on peut qualifier d'externes, et donc plus vulnérables, elles voient s'ouvrir à travers le thème de la responsabilité sociétale l'opportunité d'une reconnaissance étendue de la légitimité de leurs finalités et de leurs modalités d'action. Cela exige toutefois qu'elles réactualisent et clarifient en tant que mouvement social et politique les spécificités de leurs orientations. Ainsi, la question des responsabilités de l'économie sociale et solidaire reboucle en fin de compte sur ce qu'il est convenu d'appeler l'identité de celle-ci. Loin de clore le débat, ce colloque a le mérite de faire émerger des questionnements et des interrogations qui nous semblent indispensables.

Le comité organisateur du colloque

\section{Communications au colloque}

- Jean-Robert Alcaras et Nathalie Dompnier: Les acteurs français de l'ESS produisent-ils aujourd'hui des représentations spécifiques de la responsabilité des entreprises?

- Joëlle Argot, Hervé Daval et Jacques Poisat: Les sociétés coopératives au service du développement local.

- Lionel Arnaud: Les associations culturelles au risque du "néo-communautarisme"? Politiques de développement urbain et valorisation des liens domestiques en France et en Angleterre.

- Geneviève Azam: ESS et relocalisation de l'économie: un enjeu pour la solidarité, un enjeu pour l'écologie.
- Laurie Beurion: Responsabilités des établissements médico-sociaux et changements au regard de l'avènement des politiques sociales.

- Jérôme Blanc: L'ESS face à la responsabilité sociale des entreprises.

- Yves Bonny: Les trois sociétés civiles et la question de la responsabilité.

- Sophie Boutillier: La création d'entreprise et le rôle des structures d'accompagnement dans la réussite des projets entrepreneuriaux: éléments de réflexion à partir d'une enquête de terrain.

- Gilles Caire: Les mutuelles face à leur responsabilité d'utilité sociale: la construction du parcours de santé mutualiste. 
- Marius Chevallier: Les organisations de l'ESS peuvent-elles assumer la responsabilité d'assurer la transmission et le renouvellement des patrimoines? - Pénélope Codello-Guijarro: La responsabilité sociale: une question de qualité des services.

- Josette Combes: La construction de la responsabilité sociale, le rôle de l'éducation.

- Simon Cornée: Au-delà de la nécessité de considérer les performances sociales dans la microfinance: une proposition d'évaluation.

- Eric Dacheux et Daniel Goujon: La responsabilité épistémologique de l'économie solidaire: définir l'économie pour conforter l'économie solidaire.

- Pascale Delille: Les monnaies complémentaires, outils de régulation territoriale, pour une coresponsabilité des acteurs économiques.

- Hack-Polay Dieudonné: Compassionate leadership in voluntary organisations and the integration of forced migrants.

- Nick Dolezal et Alex Murdock: How a social economy organisation promoting festivals and selling beer contributes to a wider vision of social inclusion and solidarity? The case of the workers Beer Company in the UK.

- François Doligez: Libéralisation financière et exclusion bancaire: quelles perspectives et quelles régulations pour la microfinance dans les pays $d u$ Sud.

- François Doligez et Cécile Lapenu: Mesure des performances sociales et gouvernance des institutions de microfinance.

- Gerlinde Doyen: Les relations entre l'économie sociale belge et les pays du Sud.

- Annie Dussuet, Erika Flahault et Dominique Loiseau: Des paradoxes à linnovation dans les $O E S$ : progrès social en porte-à-faux?

- Bénédicte Fonteneau: Protection sociale en Afrique: contribution de l'économie sociale.

- Bruno Frere: L'économie solidaire entre cohérence morale et dissonance politique: un exemple de "dispute " autour du secteur des échanges sans argent (Sel et RERS). - Patrick Guiol et Jorge Muñoz: Pratiques managériales solidaires et gouvernances participatives: effets induits sur les salariés.

- Gaël Henaff: Le dirigeant d'association est-il soumis à un régime spécifique de responsabilité? - Eugène Kamalan: La responsabilité dévoyée des institutions de microfinance, évidence au Bénin.

- Anne Labit: Le partage de pouvoir au cour du projet de l'ESS.

- Rosinha Machado Carrion: Governance challenges to the consolidation of popular and solidary economy: the case of the centre for the trading of recycled materials en Porto Alegre, Brazil.

- Fayçal Mohkotary et Faouzi Tchiko: Les responsabilités de l'économie sociale et solidaire dans un pays en transition: cas de l'Algérie.

- Sambou Ndiaye: Place de l'entrepreneuriat communautaire dans la gouvernance publique locale en contexte de précarité: une expérience sénégalaise.

- Maurice Parodi: La démarche qualité, un outil d'autoévaluation ajusté à la gouvernance des entreprises sociales.

- Florian Perret: Une régulation de l'hybridation entre marchand et non-marchand: le cas des formes de production de logiciels libres.

- Antoine Pillet: Pour une adaptation critique d'outils de marketing: commercer dans le respect des valeurs d'une économie sociale et solidaire.

- Yann Régnard: Gouvernance responsable et mutualité: quelle légitimité pour les mutuelles de santé.

- Michel Rombaldi: Economie sociale et solidaire et politiques de développement territorial: nouveaux enjeux, nouvelles perspectives.

- Marie-Thérèse Taupin et Pascal Glémain: Quelle(s) responsabilité(s) pour les finances solidaires? - Guillaume Van Wijk: L'économie solidaire brésilienne et l'enjeu de la promotion d'un autre paradigme de développement. 